\title{
Permittivity Measurement of Low-Loss Substrates Based on Split Ring Resonators
}

\author{
Jianyi Li \\ 92493 Troops, Huludao, China \\ Email:dj19900805@zju.edu.cn
}

How to cite this paper: Li, J.Y. (2017) Permittivity Measurement of Low-Loss Substrates Based on Split Ring Resonators. World Journal of Engineering and Technology, 5, 62-68

https://doi.org/10.4236/wjet.2017.54B007

Received: September 3, 2017

Accepted: October 9, 2017

Published: October 12, 2017

\begin{abstract}
In this paper, we present the complex permittivity measurement of low-loss substrates based on a microstrip-line-excited split-ring resonator (SRR). Permittivity of an unknown substrate is calculated based on the change in oscillation frequency of SRR caused by the material-under-test (MUT) above the SRR. Theoretical analysis and results of the simulations and experiments demonstrate the microstrip-line-excited SRR can be used to effectively improve measurement sensitivity. Simple equations for measurement of low-loss substrates using SRR are proposed and experimentally verified.
\end{abstract}

\section{Keywords}

Split-Ring Resonators (SRR), Permittivity, Non-Contact Measurement

\section{Introduction}

Complex permittivity of the material is an important parameter to reflect the interactions of matter and magnetic fields and accurate measurement of complex permittivity is widely applied in fabrication process, quality control and biosensing.

Many methods have been proposed to measure the complex permittivity such as free-space methods, transmission-line methods and resonance methods. Free-space methods are based on the measurement of the free-space reflection and transmission coefficients of the sample placed between the antennas. This method is nondestructive and contactless; however it is more suitable for frequency higher than $30 \mathrm{G}$ and the diffraction effects around the sample edges are difficult to eliminate [1] [2] [3]. Compared to the free-space methods, transmission-line methods are much cheaper and more suitable for wideband measurements. The most common structures of transmission-line methods are rectangular waveguide and coaxial 
line [4] [5] [6]. The resonance methods are the most accurate methods to measure the complex permittivity by measuring the shift in the resonance frequency and the change in the quality factor. The resonance method offer high accuracy but have a limited bandwidth [7] [8].

In this paper, we present a non-invasive permittivity measurement of low-loss substrates based on a microstrip-line-excited split-ring resonator (SRR). Permittivity of an unknown substrate is calculated based on the change in oscillation frequency of SRR caused by the material-under-test (MUT) above the SRR. The microstrip-line-excited SRR has small size and low require of the sample's shape. Theoretical analysis and results of the simulations and experiments demonstrate the new sensor can be used to effectively improve the measurement sensitivity.

\section{Theory}

The corresponding equivalent circuit is shown in Figure 1 [9]. Based on circuit theory, the input impedance for the SRR without the SUT is

$$
Z_{0}=R_{\text {ring }}+j \omega_{0} L_{\text {ring }}+1 / j \omega_{0} C_{\text {ring }}
$$

The relationship between the impedance perturbation and the SUT's permittivity has been well investigated in many papers [10]. Assume the SRR carries a current with a magnitude $I$, the impedance perturbation caused by the SUT is

$$
\Delta Z_{L}=\left[(j \omega) / I^{2}\right] \cdot \iiint_{V} \bar{E}_{\text {inc }}(\bar{r}) \cdot\left[\left(\tilde{\varepsilon}_{r}-1\right) \cdot \bar{E}_{\text {tot }}(\bar{r})\right] d V
$$

where $\bar{E}_{\text {inc }}$ and $\bar{E}_{\text {tot }}$ are the electric fields with the SUT and without the SUT. $\bar{E}_{\text {inc }}$ denotes the electric field induced by the resonance current $I$ in the load coil, and $\bar{E}_{\text {tot }}$ denotes the total field. Thus the input impedance for the SRR with the SUT is

$$
Z=R_{\text {ring }}+j \omega L_{\text {ring }}+1 / j \omega C_{\text {ring }}+\Delta Z_{L}
$$

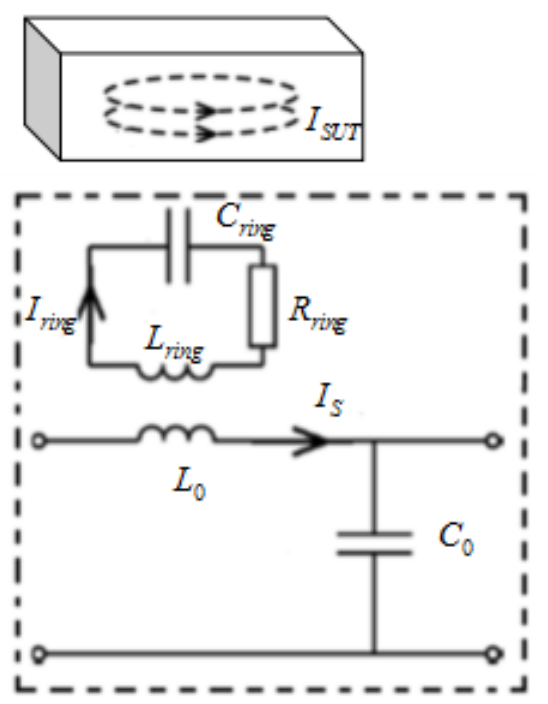

Figure 1. Equivalent circuit model for the non-contact measurement configuration of the split-ring resonator. 
Equation (2) is exact for the change in impedance due to SUT perturbations, but is not in a very usable form since we generally do not know $\bar{E}_{\text {tot }}$. So the relationship between $\Delta Z_{L}$ and $\tilde{\varepsilon}_{r}$ is not intuitive, however we can make some approximation in some restrictions.

\section{1) Contact methods}

In this section, we provide a simple setup for the contact measurement with high sensitivity, as in Figure 2. If the permittivity of the SUTs is small $\left(\varepsilon_{r} \leq 10\right)$, then we can approximate the $\bar{E}_{\text {tot }}$ by the original fields $\bar{E}_{i n c}$. Equation (2) can be simplified to

$$
\Delta Z_{L} \approx j \alpha \cdot\left(\tilde{\varepsilon}_{r}-1\right)
$$

Substituting (4) into (3), we can get

$$
Z=R_{\text {ring }}+j \omega L_{\text {ring }}+1 / j \omega C_{\text {ring }}+j \alpha\left(\widetilde{\varepsilon_{r}}-1\right)
$$

For a given configuration, $\alpha$ is a pure real constant. When the SRR resonates, imaginary part of the impedance is zero. The SUTs we measured are low-loss substrates, so we can approximate the complex permittivity ${\widetilde{\varepsilon_{r}}}^{\text {by }} \varepsilon_{\varepsilon_{r}}$ (the real part of $\underset{\varepsilon_{r}}{\sim}$ ). According such conditions, we can achieve

$$
\begin{gathered}
\omega_{s} L_{\text {ring }}-1 / \omega_{s} C_{\text {ring }}+\alpha\left(\varepsilon_{r}-1\right)=0 \\
\omega_{0} L_{\text {ring }}-1 / \omega_{0} C_{\text {ring }}=0
\end{gathered}
$$

where $\omega_{s}$ is the resonant frequency of the SRR with SUT and $\omega_{0}$ is the resonant frequency without SUT. Substituting (7) into (6), we can obtain

$$
\Delta 1 / f^{2} \approx \mathrm{A}\left(\varepsilon_{r}-1\right)
$$

\section{2) Non-contact methods}

In theory, for all the setup configuration the impedance perturbation is obtained by (2). In order to simplify (2), a contactless measurement for low-loss and small size SUT is provided, as in Figure 3. Considering the size of the SUT is much smaller than the corresponding wavelength, the Rayleigh approximation can be used to calculate the total field

$$
\bar{E}_{\text {tot }}(\bar{r}) \approx\left[3 /\left(\tilde{\varepsilon}_{r}+2\right)\right] \cdot \bar{E}_{\text {inc }}(\bar{r})
$$

Substituting (9) into (2), we get

$$
\Delta Z_{L, \text { Rayleigh }} \approx j \beta \cdot\left[\left(\tilde{\varepsilon}_{r}-1\right) \cdot\left(\tilde{\varepsilon}_{r}+2\right)\right]
$$

Then, we can also obtain a linear equation

$$
\Delta 1 / f^{2} \approx B\left(\varepsilon_{r}-1\right) /\left(\varepsilon_{r}+2\right)
$$

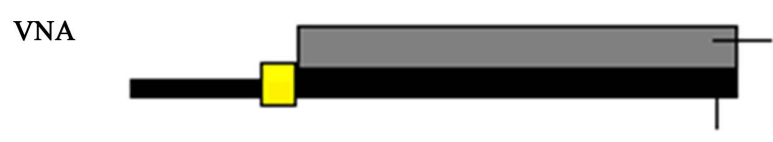

SUT

SRR

Figure 2. A typical setup of contact measurement. 


\section{Experiment}

In this section, we use two setups to measure the permittivity of the SUTs, one is the contact measurement for high sensitivity, the other is the contactless one. The microstrip-line-excited SRR is fabricated using printed circuit board technology. The implementation of the sensor is shown in Figure 4. CST microwave studio is used to simulate the mode and a VNA (AnritsuS331E) is used to measure the resonate frequency. Figure 5 shows the simulation results and the measured reflection coefficients of the SRR without SUTs. The divergence between the simulation and measurement is mainly due to errors in the fabrication process. Four substrates with the same size were prepared for the permittivity measurements.

Figure 6 shows the contact measurement result when the SUT changes and Figure 7 shows the contactless measurement results. The resonant frequency reduces as the sample permittivity increases in agreement with the theoretical analysis and simulation results.

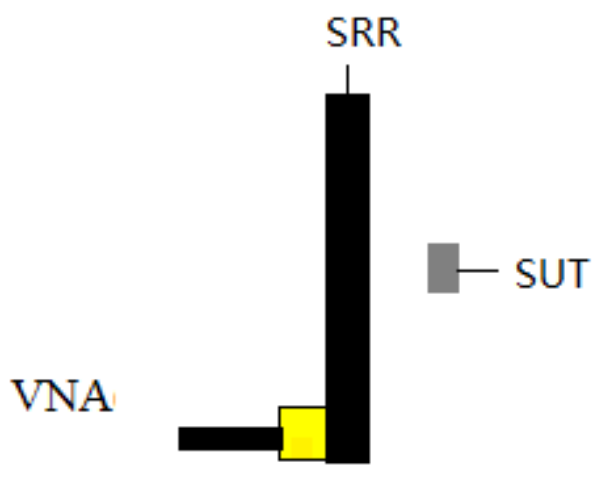

Figure 3. A typical setup of non-contact measurement.

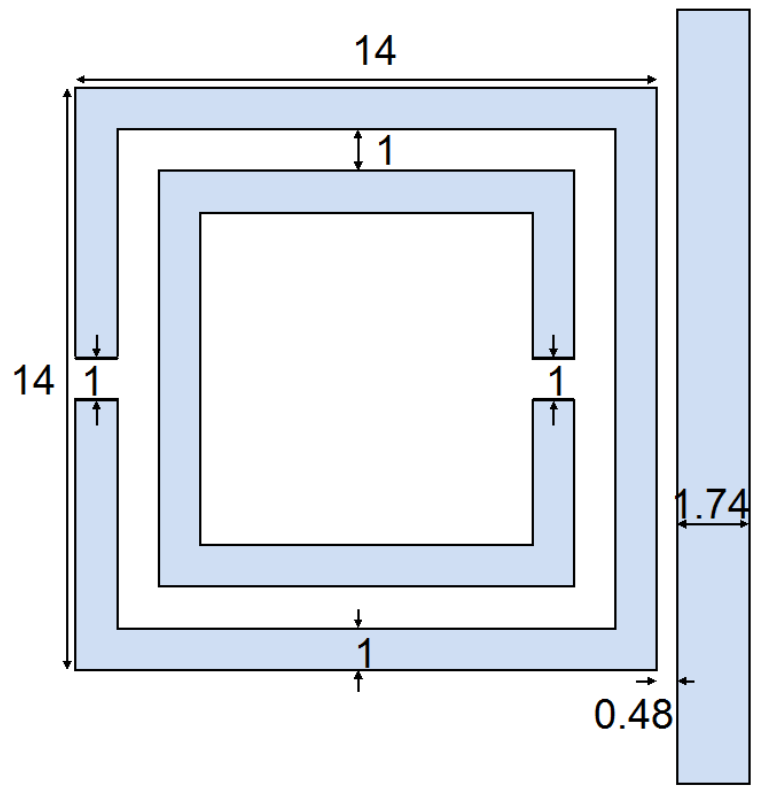

Figure 4. Implementation of the microstrip-line-excited split-ring resonator. 


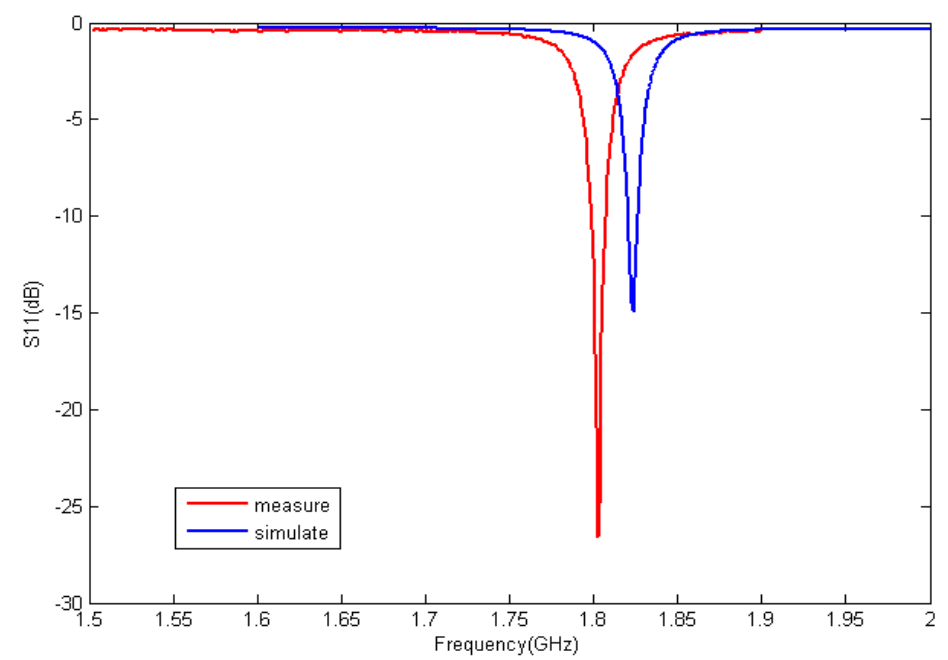

Figure 5. S-parameters of the microstrip-line-excited split-ring resonator without SUTs.

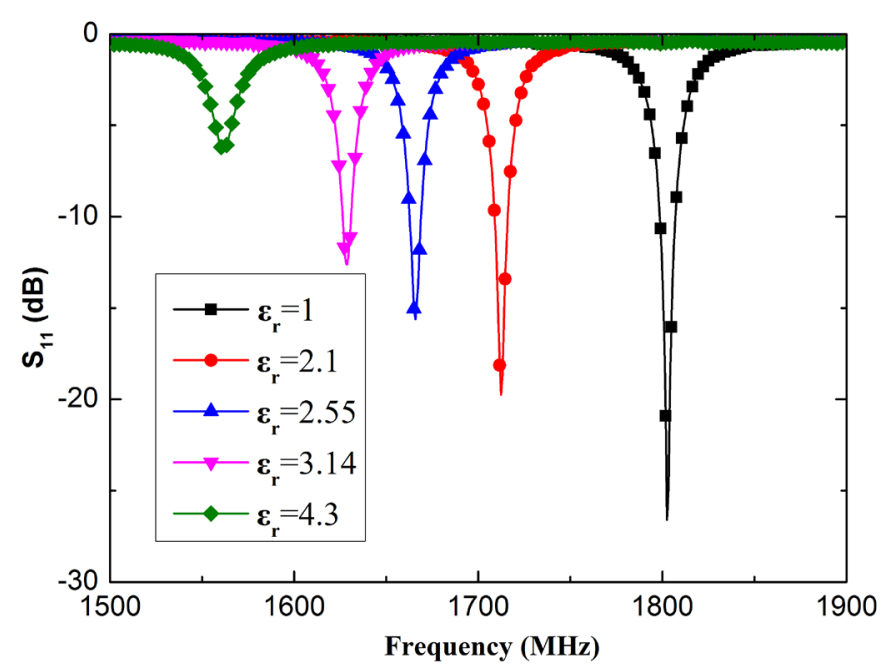

(a)

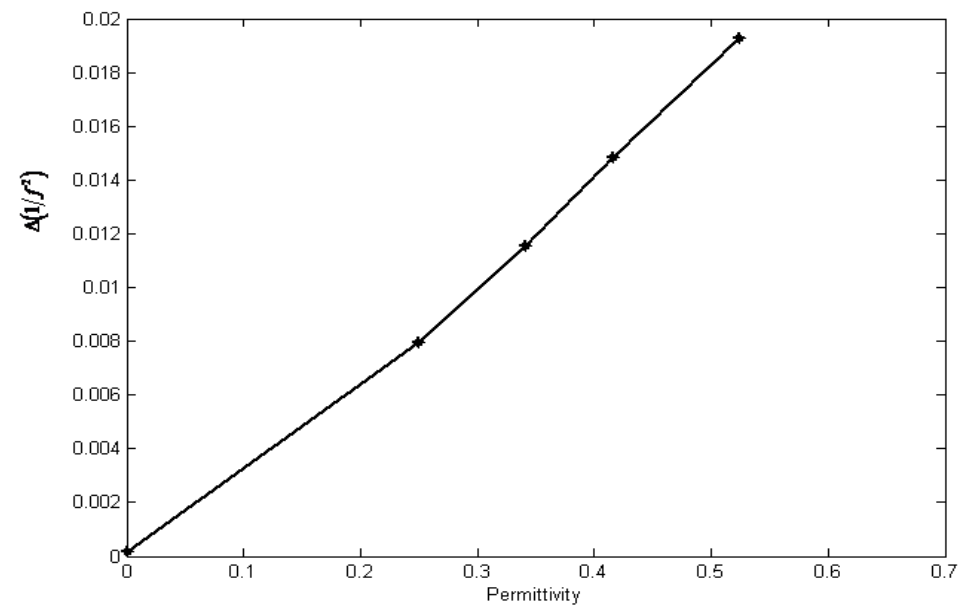

(b)

Figure 6. (a) Contact measured $S_{11}$ for different SUTs; and (b) The measured frequency with respect to permittivity. 


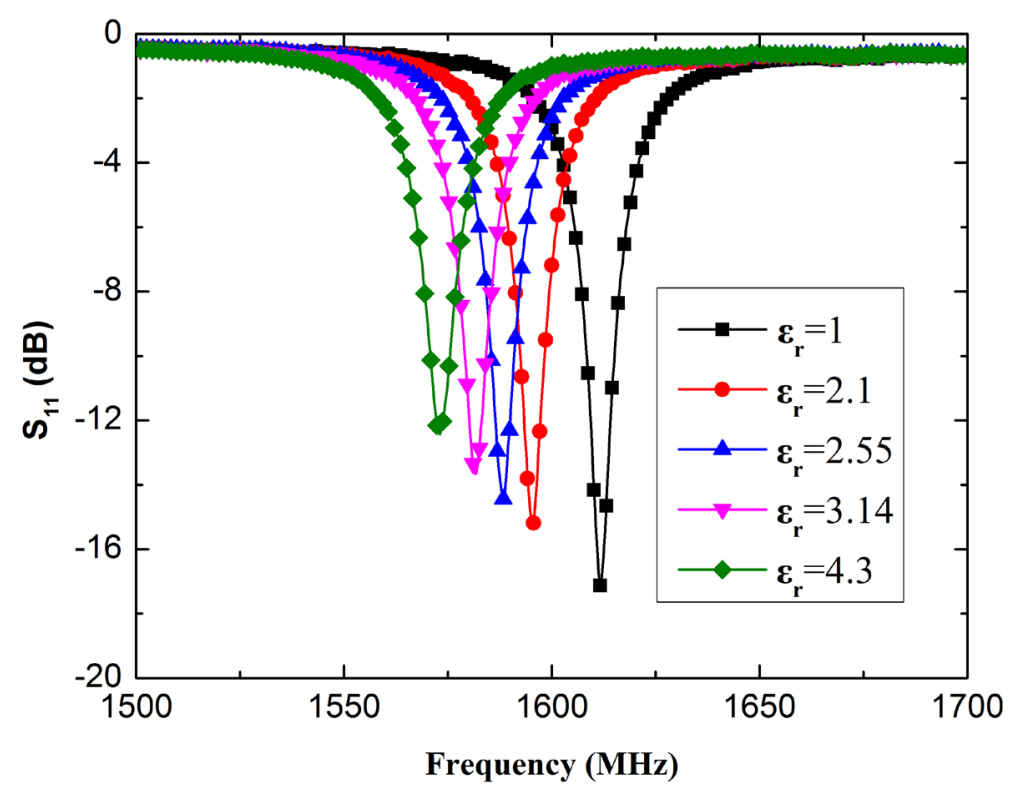

(a)

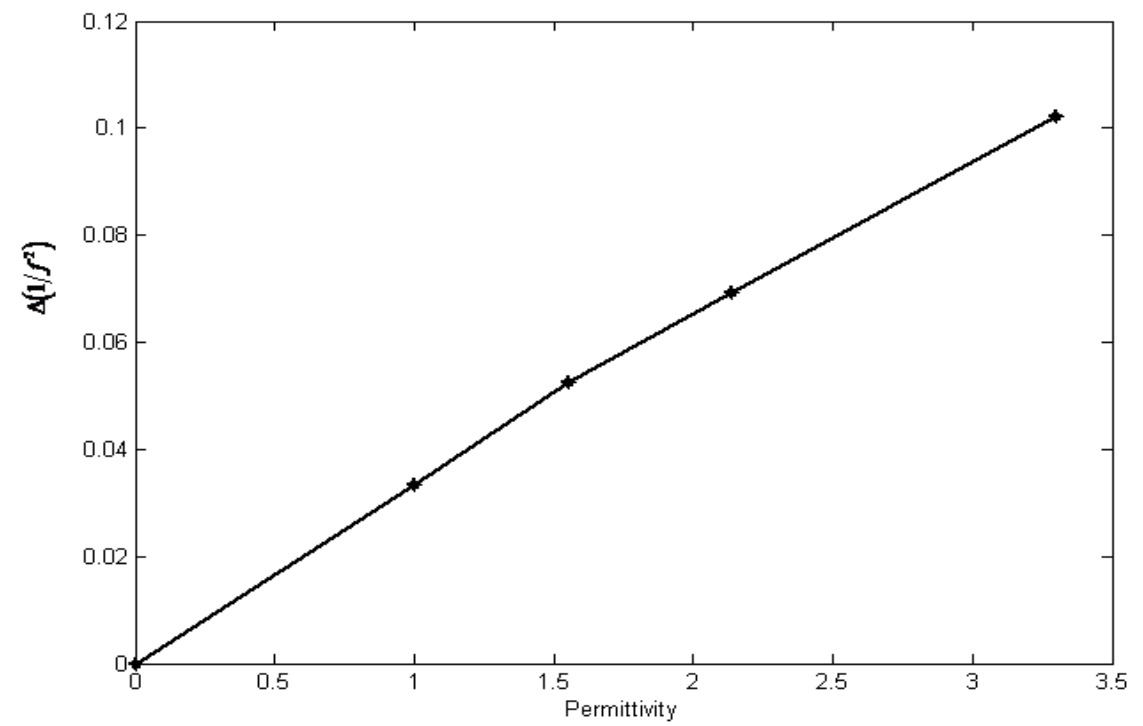

(b)

Figure 7. (a) Contactless measured $S_{11}$ for different SUTs; and (b) The measured frequency with respect to permittivity.

\section{Conclusion}

In conclusion, the theoretical analysis and results of the simulations and experiments demonstrate the microstrip-line-excited SRR can be used to effectively improve the measurement sensitivity. Simple equations for measurement of low-loss substrates using SRR are proposed and experimentally verified.

\section{References}

[1] Friedsam, G.L. and Biebl, E.M. (1997) A Broadband Free-Space Dielectirc Properties Measurement System at Millimeter Wavelengths. IEEE Trans. Instrum. Meas., 46. 
[2] Kadaba, P.K. (1984) Simultaneous Measurement of Complex Permittivity and Permeability in the Millimeter Region by a Frequency-Domain Technique. IEEE Trans. Instrum. Meas., IM-33, 336-340. https://doi.org/10.1109/TIM.1984.4315236

[3] Nitsche, R.G. and Biebl, E.M. (1994) A Free-Space Techniqque for Measuring the Complex Permittivity and Permeability in the Millimeter Wave Range. 1994 IEEE MTT-S. Microwave Symp. Dig., 1465-1468.

[4] Chin, T.C. (2005) Permittivity Measurement Technique for a Dielectric Strip Using a Rectangular Waveguide. Proc. IEEE Int. Symp. Antennas Propagat. Soc., 3, 314-317.

[5] Blackham, D.V. and Pollard, R.D. (1997) An Improved Technique for Permittivity Measurements Using a Coaxial Probe. IEEE Trans. Instrum. Meas., 46. https://doi.org/10.1109/19.676718

[6] Kehn, M.N.M., Shafai, L., Safari, F. and Noghanian, S. (2009) Permittivity Measurement of Disk and Annular Dielectric Samples Using Coaxial Transmission Line Fixtures. Part II: Experimentation and Accuracy Analyses. Can. J. Elect. Comput. Eng., 34.

[7] Meng, B., Booske, J. and Cooper, R. (1995) Extended Cavity Perturbation Technique to Determine the Complex Permittivity of Dielectric Materials. IEEE Trans. Microw. Theory Tech., 43, 2633-2636. https://doi.org/10.1109/22.473190

[8] Li, S., Akyel, C. and Bosisio, R.G. (1981) Precise Calculation and Measurements on the Complex Dielectric Constant of Lossy Material Using Cavity Perturbation Techniques. IEEE Trans. Microw. Theory Tech., MTT-29, 1041-1048.

[9] Baena, J., Bonache, J., Martin, F., Sillero, R., Falcone, F., Lopetegi, T., Laso, M., Garcia-Garcia, J., Gil, I., Portillo, M. and Sorolla, M. (2005) Equivalent Circuit Models for Split-Ring Resonators and Complementary Split-Ring Resonators Coupled to Planar Transmission Lines. IEEE Trans. Microw. Theory Tech., 53, 1451-1461. https://doi.org/10.1109/TMTT.2005.845211

[10] Auld, B.A. and Moulder, J.C. (1999) Review of Advances in Quantitative Eddy Current Nondestructive Evaluation. J. Nondestruct. Eval., 18, 3-36.

https://doi.org/10.1023/A:1021898520626 